\title{
GOBERNANZA MULTIFIDUCIARIA DE STAKEHOLDERS: ANÁLISIS COMPARADO DE LA EFICIENCIA DE BANCOS Y \\ CAJAS DE AHORROS
}

\author{
POR \\ Jose TORRES PRUÑONOSA ${ }^{1}$ \\ Jose Luis RETOLAZA ${ }^{2}$ \\ Leire SAN-JOSE ${ }^{3}$
}

\section{RESUMEN}

Las cajas de ahorros españolas son posiblemente el caso más significativo a nivel mundial de entidades multistakeholders, no sólo por su misión, sino también en su gobernanza. La Teoría Multifiduciaria de los Stakeholder plantea, al menos de forma teórica lo que en las cajas de ahorros ha sido una realidad por más de un siglo. Así, las cajas se convierten en un "caso de laboratorio" para analizar la viabilidad e idoneidad de una gobernanza participada por el conjunto de stakeholders de la organización. Nuestra hipótesis es que la gobernanza multistakeholder, así como la orientación hacia los stakeholders, generan un planteamiento estratégico diferente, no centrado en los beneficios. Por tanto, la gobernanza multifiduciaria puede perfectamente ser más ineficiente en relación con indicadores económico-financieros referidos a los shareholders de una entidad. Sin embargo, estas entidades deberían ser más eficientes si se tienen en cuenta indicadores relacionados con la generación de valor social. Con el fin de contrastar la eficiencia relativa hemos utilizado la población de bancos y cajas de España y los datos de 2009, utilizando la técnica de Análisis

\footnotetext{
${ }^{1}$ Escola Universitaria Euncet Unnim (EUNCET). Dirección de correo electrónico: jtorres@euncet.es

${ }^{2}$ Instituto de Economía Aplicada a la Empresa. [UPV/EHU]. AURKILAN Institute for Business Ethics Research. Spain. Dirección de correo electrónico: joseluis.retolaza@ehu.es

${ }^{3}$ Profesora Titular de la Universidad del País Vasco. Bilbao. Visiting Research Fellow at University of Huddersfield. United Kingdom. Dirección de correo electrónico: leire.sanjose@ehu.es
}

REVESCO Nº 108 - Segundo Cuatrimestre 2012 - ISSN: 1885-8031 - www.ucm.es/info/revesco

DOI: 10.5209/rev_REVE.2012.v18.39591

Fecha de recepción: 26/10/2011

Fecha de aceptación: 27/01/2012 
Envolvente de Datos con objeto de comparar la eficiencia económica, social y global de ambos tipos de entidades. Los resultados indican una diferenciación significativa entre estos dos grupos de entidades financieras en relación con la eficiencia social y económica, si bien no ocurre lo mismo cuando se utiliza la eficiencia global -económica más social-. Por lo que podemos concluir que la eficiencia en la gestión entre inputs y outputs en cajas de ahorros y bancos es similar.

Palabras Clave: Entidades Financieras, Teoría de Stakeholder, Valor, Eficiencia Económica, Eficiencia Social, Análisis DEA.

\section{Claves Econlit: A13, G21, P13}

\section{MULTIFIDUCIARY GOVERNANCE OF STAKEHOLDERS: A COMPARATIVE ANALYSIS OF THE EFFICIENCY OF BANKS AND SAVING BANKS}

\section{ABSTRACT}

Probably, Spanish savings banks are the most significant worldwide multistakeholders entities, not only because of their mission, but also in their governance. The stakeholder multifiduciary theory, establishes at least theoretically what the saving banks has been a reality for more than a century. Then, saving banks become "a laboratory case" to analyze the viability and eligibility of governance that is shared by all stakeholders of the organization. Our hypothesis is that multistakeholder governance as well as stakeholder orientation generates a different strategic approach, not focused on the benefits. Therefore, the multifiduciary governance may perfectly be inefficient in relation to economic and financial indicators relating to the shareholders of an entity. However, these entities should be more efficient if has been taken into account indicators related to the generation of social value. To compare the relative efficiency we have used the population of Spanish traditional and saving banks and the 2009 data, using the technique of Data Envelopment Analysis in order to compare the economic, social and global efficiency of both types of entities. The results indicate a significant differentiation between these two groups of financial institutions in relation to social and economic efficiency, but it is not happen when using the global 
efficiency-economic plus social-. So, we can conclude that the efficiency in the management between inputs and outputs in banks and savings banks is similar.

Keywords: Financial Entities, Stakeholder Theory, Value, Economic Efficiency, Social Efficiency, DEA analysis.

\section{INTRODUCCIÓN}

La viabilidad económica de las cajas de ahorros es cuestionada en la actualidad (Beltran, Torres y Prado, 2009) también la incentivación desde las instituciones públicas para su transformación en entidades bancarias tradicionales. Curiosamente las cajas de ahorros son una de las pocas organizaciones, a nivel mundial, con relevancia económica cuya Gobernanza responde a un modelo multifiduciario, en lugar del modelo general imperante, basado en los derechos de propiedad de los shareholders (García-Cestona y Surroca, 2008).

En este contexto cabe preguntarse si el modelo de gobernanza multifiduciario puede ser un componente de la actual crisis de las cajas de ahorros; aunque previamente debemos analizar desde una perspectiva científica si la situación de las cajas de ahorros en su conjunto es tan ineficiente como parece desprenderse de las actuaciones públicas y de los medios de comunicación. En este sentido, no pretendemos dar respuesta al problema de solvencia, ni a la capacidad de responder ante la actual situación de crisis, sino a la existencia de diferencias significativas entre la eficiencia de los bancos y cajas de ahorros que puedan ser atribuibles a su modelo de gobernanza.

Una mayor eficiencia de los bancos con relación a las cajas de ahorros sugeriría que la gobernanza de los shareholder resultaría más eficaz que la gobernanza multistakeholder, mientras que la inexistencia de diferencias significativas en la gestión o, por supuesto, una mayor eficiencia de las cajas de ahorros abogaría por un marco de eficiencia similar entre ambos modelos.

En los últimos veinte años, el estudio de la eficiencia de las instituciones financieras ha aumentado enormemente, como revelan los datos obtenidos en la encuesta global de Berger y Humphrey (1997) en la que se revisan 130 estudios de 21 países que aplican el 
análisis de frontera. Sin embargo, se denota aún una falta de resultados concluyentes. En consecuencia, la metodología utilizada en la presente investigación, basada en el contraste de hipótesis, ha consistido en combinar el Análisis de Datos Envolventes (DEA) con un análisis comparativo de medias entre ambos grupos (Cajas y Bancos). El análisis de eficiencia ha permitido otorgar a cada entidad una puntuación del 0 al 100 que se corresponde con su eficiencia relativa en el conjunto de la población; a continuación se han comparado las medias de eficiencia de las cajas y los bancos mediante el estadístico de contraste de la $\mathrm{f}$ de Scnedecor a través de la técnica paramétrica ANOVA.

Los análisis visualizan que las cajas y los bancos pueden tener diferencia significativa en cuanto a eficiencia en función de cómo definamos la eficiencia, si lo hacemos únicamente en términos económicos o incluimos, también, variables sociales. Más significativo aún, resulta la variabilidad intragrupo en relación con todo tipo de eficiencia; así como la distribución del riesgo entre cajas y bancos.

Los resultados sugieren que el actual proceso de restructuración de las cajas en bancos, no está fundamentado en razones de eficiencia, sino más bien en intereses económicos y políticos. Y posiblemente a la debilidad jurídica de la gobernanza multistakeholders frente a la basada en los derechos de propiedad.

\section{FUNDAMENTACIÓN TEÓRICA}

La Teoría de Stakeholder (Freeman, 1984) propone que las organizaciones no deben priorizar exclusivamente los intereses de los shareholders, sino dar una respuesta equilibrada a los intereses del conjunto de los stakeholders; siendo responsabilidad de los directivos el buscar una satisfacción equilibrada de todas las partes implicadas. A efecto del presente trabajo vamos a soslayar el debate sobre si los stakeholders se deben definir en función de su poder o de su afección por parte de la empresa; así como las diversas perspectivas de la propia Teoría: instrumental, moral, ontológica, etc.

En este sentido, Goodpaster (1991) plantea una paradoja, basada en la Teoría de Agencia que viene a decir: "Si los directivos son agentes fiduciarios de los shareholders (principal), éstos no están legitimados (ni moral ni legalmente) para mejorar las condiciones 
contractuales que estarían dispuestos a ofrecerles los shareholders de la empresa. Por lo tanto, los directivos no están legitimados para buscar la satisfacción de los intereses del conjunto de stakeholders, salvo instrumentalmente, es decir, que sea en beneficio de los shareholders".

Como respuesta teórica a este problema se genera la Teoría Multifiduciaria (Boatrigh, 1999), debido a que plantea que el problema de agencia se resuelve considerando que el principal no agrupa sólo a los shareholders, sino también al conjunto de stakeholders. Así el agente sería fiduciario del conjunto de los stakeholders, y por tanto, estaría no sólo legitimado, sino obligado a responder a los intereses de todos ellos.

En sucesivos escritos Jensen $(2002,2008)$ perfila lo que podríamos denominar como "el problema de ingobernanza", destacando que la Teoría Multifiduciaria hace ingobernable la empresa, por cuanto que no hay nadie legitimado para controlar al agente. Habida cuenta que los que deberían controlarle (principales multistakeholders) tienen intereses muy diversos entre ellos, por lo que en realidad habría un traslado del poder del principal al agente, el cual podría actuar exclusivamente en beneficio propio, sin posibilidad de control por parte del resto de los stakeholders.

En el marco de este debate Boatright (2008) plantea que en las empresas orientadas a los shareholders, el conflicto de intereses entre los mismos y la ineficacia de los instrumentos de control, impide el control del agente por parte del principal; por ende, el planteamiento multifiduciario no incrementaría el problema.

Independientemente de la perspectiva teórica que se adopte, la legislación vigente basada en los derechos de propiedad inherentes a la aportación de capital, los únicos derechos que reconoce legalmente, o por lo menos los únicos con derecho a tomar decisiones estratégicas en la empresa son los de los accionistas.

Es difícil encontrar ejemplos de funcionamiento de la Teoría Multifiduciaria que permitan un contraste empírico sobre su posibilidad y eficiencia; sin embargo, las cajas de ahorros son un caso único de aplicabilidad de este planteamiento; tanto por su dimensión económica -el total de sus activos supuso el 87,07\% del total de los activos de la banca en el año 2009-, como por su características legales (Ley 31/1985, de 2 de agosto, de regulación de 
las normas básicas sobre Órganos Rectores de las cajas de ahorro). Ya que las cajas de ahorro españolas encuentran, por una parte, claramente limitados los derechos del capital, y por otra, deben obligatoriamente incluir en sus órganos de máxima dirección a representantes del conjunto de stakeholders. Esta realidad supone un caso de suficiente entidad económica, diversidad y antigüedad como para analizar las posibilidades y problemas del modelo que nos ocupa.

Las diferencias en la estructura de propiedad, y por consiguiente, en la gobernanza de las cajas de ahorros provocan diferencias en cuanto a sus prioridades en comparación con otras entidades financieras con diferente estructura. En este sentido, las organizaciones multiobjetivo - multistakeholder, y en particular las cajas de ahorros españolas, no deben ser evaluadas exclusivamente en términos de beneficios (o costes); debido a que persiguen objetivos que se diferencian de aquellas entidades centradas en los shareholders (GarcíaCestona y Surroca, 2008).

En este contexto, existen estudios que han analizado la situación de las cajas de ahorros. La literatura financiera ha estudiado éstos aspectos desde un sentido restringido de obtención de beneficios. Así, Kumbhakar et al. (2008) analizan la eficiencia técnica de las cajas de ahorros españolas durante el período 1986-1995 y concluyen que éste se ve disminuido a lo largo del periodo, si bien encuentran evidencia de un incremento en la productividad de las cajas de ahorros en España. Otros autores como Tortosa-Ausina et al. (2002) han estudiado también la eficiencia en las cajas de ahorro españolas. Pero éstos lo han hecho para el período 1992-1998. Como en este trabajo estos autores han utilizado la técnica de análisis de eficiencia (frontera) DEA. En cuanto a los índices de productividad, las conclusiones que emergen de su estudio es que existe un crecimiento de la productividad debido a una mejora de las posibilidades de producción. En referencia a la eficiencia concluyen que la media técnica de la eficiencia es muy alta, y no varía mucho a lo largo del período analizado. Sin embargo, parece que existen diferencias significativas entre las cajas. Sus resultados son coincidentes con los obtenidos por Pastor (1995), pero difieren de los obtenidos por Grifell-Tatjé y Lovell (1996); principalmente por la elección de outputs heterogéneos y por analizarse un período diferente de la misma muestra. 


\section{PROBLEMA, HIPÓTESIS Y METODOLOGÍA}

El problema que nos ocupa es analizar si existen evidencias de que la gobernanza multistakeholder afecta negativamente a la eficiencia de una entidad financiera. Para resolver el problema hemos recurrido al contraste de hipótesis, en el ámbito del método hipotéticodeductivo; de forma previa, a fin de identificar los componentes del problema y trasladarlos a un sistema de inputs y outputs hemos recurrido al método analítico sintético.

La hipótesis fundamental $\left(\mathrm{H}_{1}\right)$ se puede enunciar como sigue: "Existe diferencia significativa entre las cajas de ahorro y los bancos en relación con su Eficiencia Global”. A fin de realizar un análisis más exhaustivo dicha hipótesis se ha subdividido en dos subhipótesis, a saber:

$\left(\mathrm{H}_{1} \mathrm{a}\right)$ "Existe diferencia significativa entre las cajas de ahorro y los bancos en relación con su Eficiencia Económica”

$\left(\mathrm{H}_{1} \mathrm{~b}\right)$ "Existe diferencia significativa entre las cajas de ahorro y los bancos en relación con su Eficiencia Social”

Para contrastar la hipótesis precedente y sus dos subhipótesis correspondientes, es necesario, por una parte, el poder identificar la población de bancos y cajas de ahorros de España y ser capaces de diferenciarlas en estas dos categorías. Para esto hemos recurrido al Anuario Estadístico de la Banca en España del año 2009 editado por la Asociación Española de Banca (AEB) y al Anuario Estadístico de las cajas de ahorros también del 2009 editado por la Confederación Española de Cajas de Ahorros (CECA). Hay que señalar que se han quedado fuera del estudio las Cooperativas de crédito, que si bien son un modelo financiero sumamente interesante, a nivel de la Teoría Multifiduciaria representan un planteamiento intermedio [plurifiduciario], por lo que su posible relación con la eficiencia no resulta tan evidente a la hora de falsar el "problema de ingobernanza de Jensen".

En segundo lugar resulta imprescindible obtener una medida de la eficiencia de cada entidad financiera, para lo que se ha considerado conveniente recurrir al Análisis de Datos Envolventes (Metodología DEA). Ésta está basada en la medición de la eficiencia relativa y se remonta a Farell (1957) que desarrolla el trabajo previo de Debreu (1951) y Koopmans (1951), los cuales definen la eficiencia empresarial considerando múltiples inputs. 
Concretamente, se propone medir la eficiencia en función de dos componentes básicos; por un lado la technical efficiency que refleja la habilidad de una empresa para obtener el máximo output desde un conjunto de inputs; y por otro lado desde la allocative efficiency, que refleja la habilidad de la empresa en la utilización de los inputs en proporciones optimas y en función de sus respectivos precios. La combinación de estas dos medidas provee la medida de la economic efficiency. Asimismo, señalar que el concepto de eficiencia se define en una unidad de decisión como la posición relativa de esa unidad respecto de la frontera de la mejor ejecución, establecida matemáticamente por el ratio compuesto por la suma de los outputs frente a los inputs y sus correspondientes ponderaciones (Charnes et al., 1978).

La metodología DEA es una técnica no paramétrica determinista propuesta por Charnes et al. (1978) y desarrollada por Banker et al. (1984) y Banker (1984). Esta técnica está especialmente indicada para calcular la eficiencia de unidades productivas con múltiples outputs e inputs. Por consiguiente, su carácter no paramétrico evita la imposición de una forma funcional determinada. Esta metodología estima la frontera de producción mediante técnicas de programación lineal; ésta viene determinada por unas funciones envolventes de las combinaciones de outputs-inputs proporcionadas por los datos empíricos, para lo que se requiere disponer de información de varias unidades de decisión o decision making units (DMU) (para un desarrollo más exhaustivo de su fundamentación puede consultarse Charnes et al. [1995], y para un análisis de las metodologías empleadas en la década de los 70 y 80 que es cuando realmente adquiere importancia puede consultarse Seiford \& Thrall [1990] y Seiford [1996]). Las DMUs pueden ser empresas, unidades estratégicas de negocio, departamentos, incluso procesos específicos de las propias empresas.

En nuestro caso consideraremos a cada entidad financiera (Banco o Caja) como una DMU. El valor de máxima eficiencia es igual a 1, o a 100\%, dependiendo de la escala utilizada por el modelo DEA; en nuestro caso utilizaremos el "Analyst Frontier 4" que utiliza una escala de 100. A medida que una empresa se encuentre alejada de la frontera (viene determinada por el conjunto de unidades de decisión que obtienen la máxima eficiencia), el valor descenderá y se situará entre $100 \%$ y $0 \%$. Este método nos permite obtener la eficiencia relativa, y no la absoluta. De esta forma se obtienen las DMUs más eficientes en comparación a la selección considerada, es decir, se establecen cuáles son aquellas unidades que en comparación con el resto son más eficientes. Dicho análisis funciona mejor cuando, como en 
este trabajo, se puede realizar sobre el conjunto de la población y no sólo sobre una muestra de la misma.

Teniendo en cuenta que el análisis DEA se basa en una relación entre inputs y outputs (véase Tabla 1), la selección y cuantificación de los mismos será clave en la obtención del resultado. Han sido considerados los siguientes inputs y outputs.

Tabla 1. Inputs y Outpus.

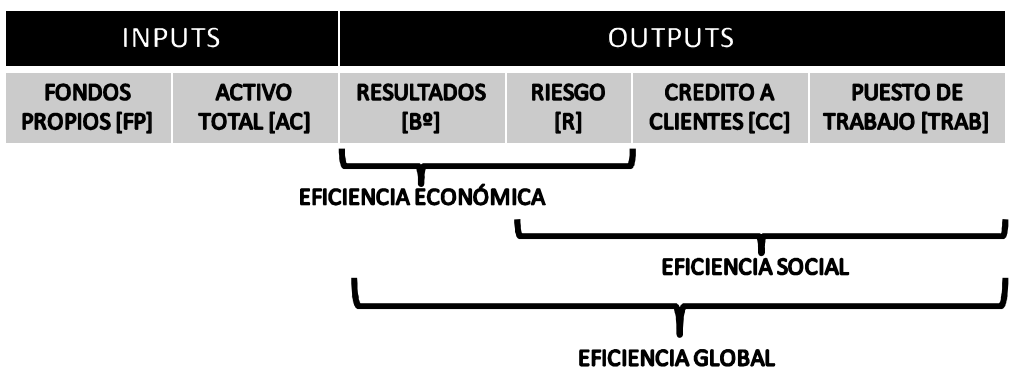

Fuente: Elaboración Propia.

Como inputs del sistema se han considerado los Fondos Propios y el Activo Total; ambas variables se consideran pertinentes por cuanto forman parte de los indicadores de rentabilidad financiera y económica respectivamente; no se ha utilizado el Fondo de Dotación fundacional ${ }^{4}$ como inputs dado que en las cajas de ahorros es irrelevante respecto a los Fondos propios y al activo, con lo cual daría lugar a eficiencias desproporcionadas.

En cuanto a los outputs se han considerado cuatro, por una parte vinculados a la eficiencia económica se han tenido en cuenta el resultado de la actividad de explotación 5 obtenido y el riesgo soportado. Dado que algunas entidades habían obtenido resultados negativos en el 2009 y que los análisis de eficiencia sólo trabajan con resultados positivos, se ha realizado un algoritmo de transformación consistente en $\mathrm{y}=\mathrm{x}+(\min . \mathrm{X}+1)^{6}$. Con relación al riesgo se ha obtenido del sumatorio de los riesgos y compromisos contingentes reconocidos por las diferentes entidades.

\footnotetext{
${ }^{4}$ El equivalente al Capital Social para un banco.

${ }^{5}$ Se ha optado por este parámetro puesto que refleja la eficiencia anual mejor que el resultado antes de impuestos, ya que las pérdidas y ganancias extraordinarias pueden distorsionar los resultados si sólo se tiene en cuenta el periodo de un año, como es el caso.

${ }^{6} \mathrm{Y}$ es la puntuación resultante; $\mathrm{x}$ es la puntuación directa obtenida por cada DMU; min x es la menor puntuación obtenida por una DMU.
}

REVESCO No 108 - Segundo Cuatrimestre 2012 - ISSN: 1885-8031 - www.ucm.es/info/revesco 
En relación con la eficiencia social, ha sido más difícil identificar posibles outputs, ya que no existe un sistema de indicadores estandarificado que mida la rentabilidad social o la rentabilidad aportada a otros grupos de stakeholders distintos a los shareholders; no obstante, de los datos aportados por las entidades hemos optado por seleccionar tres; por una parte, el número de empleos generado, por cuanto que la generación de trabajo tiene un claro valor social con impacto positivo tanto en la economía como en la recaudación impositiva. Por otra parte se ha tomado en consideración los créditos a clientes, que se han considerado un indicador incompleto de apoyo a la economía real por parte de las entidades; hubiera sido más apropiado analizar la calidad de la colocación del activo pero la mayoría de las entidades son opacas al respecto (San-Jose et al., 2011). Por último, se ha considerado como output el inverso del riesgo, también considerado en la eficiencia económica, por cuanto que se relaciona directamente con el riesgo moral asumido por las entidades y cuyo impacto social negativo se ha constatado a nivel internacional desde los primeros momentos de la crisis. En cuanto a la variable riesgo señalar que hay algunas entidades cuyo valor es 0 , a fin de poder incluirlas en el análisis se ha reemplazado dicha puntuación por 0,01 , lo cual permite operar con ellas, sin que la atribución de valor suponga un desajuste de las eficiencias relativas.

En lo que se refiere a la Obra Social de las cajas de ahorros, que habitualmente se identifican como el principal output social, hemos considerado no tenerlo en cuenta, por cuanto que una parte importante del montante destinado a la obra social se detrae de impuestos, los cuales, sin embargo sí abonan los bancos; por lo que el análisis quedaría referido a si resulta mejor la gestión pública o privada de los fondos sociales. En investigaciones posteriores resultaría interesante incluir un output que totalizara el sumatorio de la de la obra social más impuestos, ya que el resultado sería una variable numérica positiva, en la práctica totalidad de los casos, que reflejaría la aportación económica dedicada a actividad social por parte de las diferentes entidades financieras.

Las DMUs utilizadas en este artículo agrupan la totalidad de la población compuesta por 109 unidades. No obstante, de cara a los análisis de eficiencia se han descartado 10 unidades: La Caja de Castilla la Mancha, por ser la única unidad con fondos propios negativos, lo cual desvirtuaba cualquier análisis de eficiencia; el Banesto Banco de Emisiones, el BBV Banco de Financiación, el Banco Industrial de Bilbao, el Banco de Promoción de Negocios, el Banco Occidental, el Banco de Albacete, y el Banco Alicantino de 
Comercio, por no contar con trabajadores, lo que hace suponer que no se dedica a una actividad de explotación bancaria ordinaria; así como el Banco Liberta, por darse una coincidencia de los fondos propios con el activo.

A efectos del análisis DEA se han realizado 7 análisis de eficiencia, tres considerando únicamente el input de Activo Total y respectivamente los outputs de Eficiencia Económica, Eficiencia Social, Eficiencia Global; otros tres se han realizado utilizando los mismos outputs y como inputs el Activo Total y los Fondos propios conjuntamente; en este segundo grupo de análisis se ha incluido uno en el que sólo se considera el output resultado. Este último análisis no se ha realizado utilizando un único input ya que son necesarios, al menos, tres variables para poder realizar un análisis DEA.

Para el retorno de los datos se ha utilizado la escala de retornos variables (BBC) propuesta por Banker et al. (1984) que es una mejora de la comúnmente utilizada escala de retornos constantes (CCR) de Charnes et al. (1978).

\section{ANÁLISIS DE DATOS}

El listado de DMUs, así como los resultados obtenidos en el análisis de la eficiencia relativa, para los siete contrastes realizados, se adjuntan en forma de tabla en el anexo. En la siguiente figura (véase Figura 1) pueden visualizarse los resultados obtenidos para las tres hipótesis. 
Figura 1. Dispersión de la Eficiencia Económica, Social y Global entre bancos y cajas de ahorros españolas. 2009.
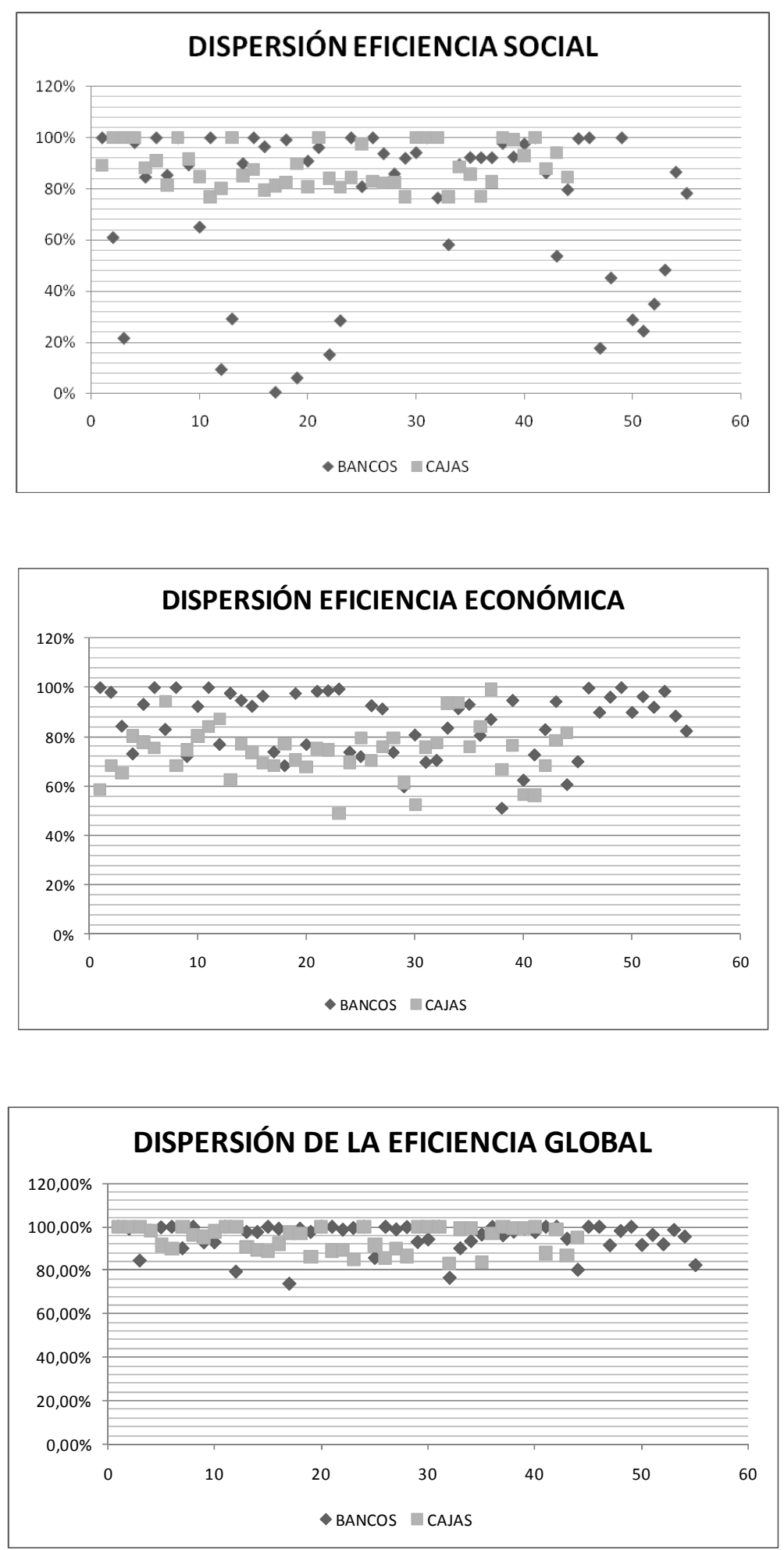

Fuente: Elaboración Propia. 
Una vez obtenidas las puntuaciones de eficiencias para cada uno de los DMUs, se ha procedido a realizar una comparación de medias mediante la técnica ANOVA, utilizando como estadístico de contraste la F de Snedecor con un nivel de significación del 99,5\%. Los resultados obtenidos han quedado representados en la siguiente tabla (véase Tabla 2):

Tabla 2. Resultados descriptivos y ANOVA.

\begin{tabular}{|c|c|c|c|c|c|c|c|}
\hline VARIABLES & \begin{tabular}{|l} 
TIPO \\
ENTIDAD
\end{tabular} & $\mathbf{N}$ & Media & $\begin{array}{c}\text { Desviación } \\
\text { Típica }\end{array}$ & Error Típico & F (Sig.) & $\begin{array}{c}\text { Estadístico de } \\
\text { Levene }\end{array}$ \\
\hline \multirow[t]{3}{*}{ 1.Beneficio_Eco_2Inputs } & Caja & 43 & 73,77 & 10,94 & 1,67 & \multirow{3}{*}{$23,463(0,000) * * *$} & \multirow{3}{*}{$3,53(0,063)^{*}$} \\
\hline & Banco & 58 & 85,87 & 12,62 & 1,66 & & \\
\hline & Total & 101 & 80,72 & 13,31 & 1,32 & & \\
\hline \multirow[t]{3}{*}{ 2. Beneficio_Eco_Riesgo_2Inputs } & Caja & 43 & 75,88 & 10,40 & 1,59 & \multirow{3}{*}{$23,484(0,000) * * *$} & \multirow{3}{*}{$3,56(0,062)^{*}$} \\
\hline & Banco & 58 & 87,92 & 11,98 & 1,57 & & \\
\hline & Total & 101 & 82,80 & 12,77 & 1,27 & & \\
\hline \multirow[t]{3}{*}{ 3. Beneficio_Social_2Inputs } & Caja & 43 & 88,33 & 8,12 & 1,24 & \multirow{3}{*}{$8,809(0,004)^{* * *}$} & \multirow{3}{*}{$51,321(0,000) * * *$} \\
\hline & Banco & 58 & 71,53 & 32,04 & 4,21 & & \\
\hline & Total & 101 & 78,68 & 26,12 & 2,60 & & \\
\hline \multirow[t]{3}{*}{ 4. Beneficio_Global_2Inputs } & Caja & 43 & 94,49 & 5,75 & 0,88 & \multirow{3}{*}{$0,390(0,534)$} & \multirow{3}{*}{$0,290(0,591)$} \\
\hline & Banco & 58 & 96,15 & 5,75 & 0,76 & & \\
\hline & Total & 101 & 95,44 & 5,78 & 0,58 & & \\
\hline \multirow[t]{3}{*}{ 5. Beneficio_Eco_Riesgo_1lnput } & Caja & 43 & 71,50 & 11,64 & 1,77 & \multirow{3}{*}{$20,186(0,000) * * *$} & \multirow{3}{*}{$2,607(0,110)$} \\
\hline & Banco & 58 & 83,12 & 13,55 & 1,81 & & \\
\hline & Total & 101 & 78,07 & 13,95 & 1,40 & & \\
\hline \multirow[t]{3}{*}{ 6. Beneficio_Social_1Input } & Caja & 43 & 89,26 & 8,35 & 1,27 & \multirow{3}{*}{$11,629(0,001)^{* * *}$} & \multirow{3}{*}{$60,077(0,000)^{* * *}$} \\
\hline & Banco & 58 & 88,26 & 22,78 & 2,99 & & \\
\hline & Total & 101 & 88,69 & 18,04 & 1,80 & & \\
\hline \multirow[t]{3}{*}{ 7. Beneficio_Global_1Input } & Caja & 43 & 93,01 & 5,69 & $\overline{0,87}$ & \multirow{3}{*}{$0,001(0,975)$} & \multirow{3}{*}{$1,350(0,248)$} \\
\hline & Banco & 58 & 93,05 & 7,79 & 1,02 & & \\
\hline & Total & 101 & 93,03 & 6,94 & 0,69 & & \\
\hline
\end{tabular}

** La varianza entre componentes es negativa. Ha sido reemplazada por 0,0 al calcular esta medida para los efectos aleatorios.

*** Significativo al $1 \%$ * *Significativo al $10 \%$

Fuente: Elaboración Propia.

Como puede apreciarse los resultados demuestran que hay diferencia significativa en los contrastes 1, 2, 3, 5 y 6, mientras se debe mantener la hipótesis nula en los contrastes 4 y 7. Sin embargo hay que tener en cuenta que la ANOVA compara las medias de grupos de similar tamaño y que presupone varianzas similares entre cada grupo.

Para comprobar que nuestra muestra cumple con la segunda premisa se ha realizado un Test de Levene (Levene, 1960) en el que se ha obtenido el resultado que se muestra en la tabla 2. Como puede observarse en el cuarto, quinto y séptimo contraste debemos aceptar la hipótesis alternativa, considerando que no hay igualdad entre las varianzas, por lo que no sería concluyente la F de Snedecor; para solucionar el problema hemos utilizado dos estadísticos complementarios, Welch y Brown-Forsythe (véase Tabla 3), los cuales aportan una mayor robustez a los análisis de igualdad de medias (Brown-Forsythe, 1974); como puede 
comprobarse los resultados de ambos estadísticos coinciden entre ellos y coinciden con los resultados obtenidos para la F de Snedecor.

Tabla 3. Pruebas robustas de igualdad de medias.

\begin{tabular}{|c|c|c|c|c|c|}
\hline VARIABLES & Técnica & Estadístico $^{\mathrm{a}}$ & gl1 & gl2 & Sig. \\
\hline \multirow[t]{2}{*}{ 1. Beneficio_Eco_2Inputs } & Welch & 26,516 & 1 & 96,521 & $0,000 * * *$ \\
\hline & Brown-Forsythe & 26,516 & 1 & 96,521 & $0,000 * * *$ \\
\hline \multirow[t]{2}{*}{ 2. Beneficio_Eco_Riesgo_2Inputs } & Welch & 29,036 & 1 & 96,492 & $0,000 * * *$ \\
\hline & Brown-Forsythe & 29,036 & 1 & 96,492 & $0,000 * * *$ \\
\hline \multirow[t]{2}{*}{ 3. Beneficio_Social_2Inputs } & Welch & 12,846 & 1 & 66,375 & $0,001 * * *$ \\
\hline & Brown-Forsythe & 12,846 & 1 & 66,375 & $0,001 * * *$ \\
\hline \multirow[t]{2}{*}{ 4. Beneficio_Global_2Inputs } & Welch & 2,077 & 1 & 90,684 & 0,153 \\
\hline & Brown-Forsythe & 2,077 & 1 & 90,684 & 0,153 \\
\hline \multirow[t]{2}{*}{ 5. Beneficio_Eco_Riesgo_1Input } & Welch & 0,094 & 1 & 76,133 & 0,761 \\
\hline & Brown-Forsythe & 0,094 & 1 & 76,133 & 0,761 \\
\hline \multirow[t]{2}{*}{ 6. Beneficio_Social_1lnput } & Welch & 14,677 & 1 & 66,63 & $0,000 * * *$ \\
\hline & Brown-Forsythe & 14,677 & 1 & 66,63 & $0,000 * * *$ \\
\hline \multirow[t]{2}{*}{ 7.Beneficio_Global_1Input } & Welch & 0,001 & 1 & 98,986 & 0,977 \\
\hline & Brown-Forsythe & 0,001 & 1 & 98,986 & 0,977 \\
\hline
\end{tabular}

a. Distribuidos en F asintóticamente.

$* * *$ Significativo al $1 \%$

Fuente: Elaboración Propia.

De los resultados obtenidos se desprende que la técnica utilizada puede no ser la correcta en el caso de los contrastes relativos a la diferencia en la eficiencia global, ya que en este caso tenemos dudas sobre la normalidad de los datos. A fin de solucionar este problema, se ha utilizado también la técnica no paramétrica de Kolmogorov-Smirnov (véase Tabla 4); que como puede apreciarse en la siguiente tabla, coincide plenamente en sus resultados con los obtenidos en el análisis ANOVA, y salva los problemas de homocedasticidad detectados con el estadístico de Levene .

Tabla 4. Estadístico de contraste $\mathrm{Z}$ de Kolmogorov-Smirnov.

\begin{tabular}{|c|c|c|c|c|c|c|c|c|}
\hline \multicolumn{2}{|c|}{ Z de Kolmogorov-Smirnov } & $\begin{array}{c}1 . \\
\text { Beneficio_Ec } \\
\text { o_2Inputs }\end{array}$ & $\begin{array}{c}2 . \\
\begin{array}{c}\text { Beneficio_Ec } \\
\text { o_Riesgo_2l } \\
\text { nputs }\end{array}\end{array}$ & $\begin{array}{c}3 . \\
\text { Beneficio_So } \\
\text { cial_2Inputs }\end{array}$ & $\begin{array}{c}4 . \\
\text { Beneficio_GI } \\
\text { obal_2Inputs }\end{array}$ & $\begin{array}{c}5 . \\
\text { Beneficio_Ec } \\
\text { o_Riesgo_1l } \\
\text { nput }\end{array}$ & $\begin{array}{c}6 . \\
\text { Beneficio_So } \\
\text { cial_1Input }\end{array}$ & $\begin{array}{c}7 . \\
\text { Beneficio_Gl } \\
\text { obal_1Input }\end{array}$ \\
\hline \multirow{3}{*}{$\begin{array}{l}\text { Diferencias } \\
\text { más } \\
\text { extremas }\end{array}$} & Absoluta & ,534 &, 534 & ,321 & ,204 & ,527 & ,339 & ,179 \\
\hline & Positiva &, 000 &, 000 & ,321 &, 089 &, 000 & ,339 & , 125 \\
\hline & Negativa &,- 534 &,- 534 &,- 117 &,- 204 &,- 527 &,- 064 &,- 179 \\
\hline \multicolumn{2}{|c|}{ Z de Kolmogorov-Smirnov } & 2,632 & 2,632 & 1,585 & 1,008 & 2,597 & 1,673 & ,885 \\
\hline \multicolumn{2}{|c|}{ Sig. asintót. (bilateral) } & $0,000^{\star \star *}$ & $0,000^{\star \star *}$ & $0,013^{* *}$ & 0,262 & $0,000^{* \star *}$ & $0,007^{* \star \star}$ & 0,414 \\
\hline
\end{tabular}

Fuente: Elaboración Propia.

REVESCO No 108 - Segundo Cuatrimestre 2012 - ISSN: 1885-8031 - www.ucm.es/info/revesco 
En resumen, los resultados obtenidos permiten obtener con relación a las hipótesis planteadas los siguientes resultados:

1. Se confirma la Hipótesis $\left(\mathrm{H}_{1} \mathrm{a}\right)$ : "Existe diferencia significativa entre las cajas de ahorro y los bancos en relación con su Eficiencia Económica”, siendo ésta mayor en las entidades bancarias.

2. Se confirma la Hipótesis $\left(\mathrm{H}_{1} \mathrm{~b}\right)$ : “Existe diferencia significativa entre las cajas de ahorro y los bancos en relación con su Eficiencia Social”, siendo ésta mayor en las cajas de ahorros.

3. Se refuta la Hipótesis $\left(\mathrm{H}_{1}\right)$ : “Existe diferencia significativa entre las cajas de ahorro y los bancos en relación con su Eficiencia Global”. En relación con la Eficiencia Global, entendida como una suma de la eficiencia económica y social, debe mantenerse la hipótesis nula, ya que no se han apreciado diferencias significativas entre bancos y cajas de ahorros.

\section{CONCLUSIONES}

La comparación realizada con datos de 2009 en cuanto a la eficiencia entre bancos y cajas utilizando la población española y mediante el análisis DEA nos llevan a determinar por un lado que los bancos sólo son más eficientes que las cajas de ahorro si se tiene en cuenta el resultado económico, pero no si se tiene en cuenta el impacto global, donde ambos tipos de entidades son equiparables. Por otro lado, y en relación con el problema de gobernanza que origina el análisis podemos señalar, que tal y como cabría esperar, las cajas son menos eficientes si se tienen en cuenta únicamente los intereses de los shareholders, siendo más eficientes en relación con el conjunto de intereses de los stakeholders, tal y como cabría esperar tanto desde la teoría como desde la composición de sus órganos rectores. La similitud de la Eficiencia Global nos llevaría a rechazar la hipótesis de ingobernanza de Jensen (Jensen, 2002, 2008; Retolaza et al., 2010), por cuanto que ambos tipos de entidades tienen unos niveles de eficiencia similares.

En este contexto, el resultado obtenido resulta significativo en referencia al problema de aplicabilidad de la Teoría de Stakeholder, y más en concreto en relación con la perspectiva Multifiduciaria. Como hemos señalado, las cajas de ahorros constituyen uno de los pocos tipos de organización donde la gobernanza no se vincula con los derechos de propiedad, e 
indudablemente es el de mayor envergadura económica tiene en España. Que la gobernanza multifiduciaria alcance niveles de Eficiencia Global similares a los de la Banca, lleva a corroborar la posibilidad de este tipo de gobernanza en entidades económicamente significativas; lo que abre una nueva perspectiva a la aplicabilidad de la Teoría de Stakeholder en su perspectiva más ontológica o constituyente, donde los derechos de los stakeholders no se mediatizan a través de la dirección, sino que encuentran su representación a través de la participación en los órganos de gobernanza de la entidad.

En relación con las últimas decisiones de bancarización de las cajas por parte del Gobierno, los resultados obtenidos llevan a cuestionar las premisas que las sustentan; ya que si bien es cierto que los resultados económicos son menores en este tipo de entidades, lo cierto es que los resultados sociales son mayores. Se ha argumentado, en general, sobre la menor solvencia de las cajas con relación a los bancos; pero dicha diferencia no resulta significativa, mientras que sí resulta significativo el mayor riesgo contingente soportado por los bancos ${ }^{7}$, aún cuando éste use la solvencia como factor corrector.

En esta línea, cabe preguntarse por qué se aplican con carácter general unas medidas a un tipo de entidades cuando de los análisis se desprende que las faltas de eficiencia y el riesgo asociado se distribuye aleatoriamente entre bancos y cajas. La respuesta quizás la encontremos de nuevo en la Teoría de los Derechos de Propiedad, ya que posiblemente resulte mucho más fácil regular un subsector (cajas de ahorro) donde no hay "propietarios" afectados negativamente y de debate se circunscribe a ámbitos políticos. Indudablemente el respetar los derechos de propiedad y no intromisión en la Banca, y a su vez, desmontar un sistema financiero que precisamente se puede proponer como una alternativa al actual sistema financiero mundial centrado en el beneficio de los shareholders y que tanto ha tenido que ver con la actual crisis en la que nos encontramos, posiblemente va a ser bien visto por los "mercados financieros"; más aún si se tiene en cuenta una parte importante del negocio de las cajas, más del 50\% del mercado financiero español, va a pasar a generar beneficios privados, lo que hasta ahora no ocurría. Lo cierto es que no es la ineficiencia en la gestión de las cajas, que por supuesto es mejorable, como la de muchos bancos, la que lleva a la depauperación del modelo por parte de los poderes públicos, sino la presión de unos mercados que no

\footnotetext{
${ }^{7}$ Se ha considerado la totalidad del riesgo dividido entre el activo total y entre la solvencia.
}

REVESCO No 108 - Segundo Cuatrimestre 2012 - ISSN: 1885-8031 - www.ucm.es/info/revesco 
comprenden el modelo, en el mejor de los casos, o que incluso lo ven como una amenaza a sus intereses especulativos. La famosa refundación del capitalismo se ha traducido más bien en una consolidación del capitalismo, eliminando un modelo que precisamente hubiera podido ser un buen modelo para su refundación.

Para finalizar señalar que las principales limitaciones de la investigación vienen dadas por la utilización de los datos de un único ejercicio y por la no existencia de unos indicadores homologados relacionados con la eficiencia social. Como futuras líneas de investigación estaría la réplica del estudio utilizando un período mayor de años; y a más largo plazo, el establecer un sistema de indicadores que permitieran cuantificar el valor generado para los diferentes stakeholders; así mismo sería interesante incluir un otput complementario que contemplara la aportación económica de las diferentes entidades a un uso social, bien mediante la obra social o la aportación de impuestos.

\section{BIBLIOGRAFÍA}

AEB: Anuario Estadísticio de la Banca en España, Diciembre 2009. Madrid: Asociación Española de Banca.

BANKER, R.: Estimating most productive scale size in data envelopment analysis. European Journal of Operational Research (17), 35-44.Banker, R., Charnes, A., \& Coopers, W. (1984). Some models for estimating technical and scale inefficiencies in data envelopment analysis. Management Science, 30 (9), 1078-1092.

BERGER, A., \& HUMPHREY, D.: Efficiency of financial institutions: International survey and directions for future research. European Journal of Operational Research (98), 175$212,1997$.

BELTRAN, J., TORRES, J., PRADO, M.: El actual proceso de fusiones de las cajas de ahorros; un análisis empírico de sus posibles causas económico-financieras. En REDONDO, J.A., Creando clientes en mercados globales. 2009, Santiago de Compostela: AEDEM.

BOATRIGHT, J.: Ethics in Finance (2nd Edition: 2008 ed.). Malden: Blackwell, 1999.

BROWN, M. B., \& FORSYTHE, A.: Robust tests for the equality of variances. Journal of the American Statistical Association (69), 364-367, 1974. 
CECA: Anuario Estadístico de las Cajas de Ahorros, 2009. Madrid: Confederación Española de Cajas de Ahorros.

CHARNES, A., COOPER, W., \& RHODES, E.: Measuring the efficiency of decision making units. European Journal of Operational Research , 429-444, 1978.

DEBREU, G.: The coefficient of resource utilisation. Econometrica (19), 273-292, 1951.

FARRELL, J.: The measurement of productive efficiency. Journal of the Royal Statistical Society (120), 253-281, 1957.

FREEMAN, R.: Strategic management: A Stakeholder Approach. 1984, Boston: Pitman.

GARCÍA-CESTONA, M., \& SURROCA, J.: Multiple goals and ownership structure: Effects on the performance of Spanish savings banks. European Journal of Operational Research , 187 (2), 582-599, 2008.

GOODPASTER, K.: Business Ethic and Stakeholder Analysis. Business Ethics Quarterly , 1 (1), 53-73, 1991.

GRIFELL-TATJÉ, E., \& LOVELL, C.: Deregulation and productivity decline: The case of Spanish savings banks. European Economic Review , 40 (6), 1281-1303, 1996.

JENSEN, M.: Non-Rational Behaviour, Value Conflicts, Stakeholder Theory, and Firm Behaviour. Business Ethics Quarterly, 18 (2), 167-171, 2008.

JENSEN, M.: Value Maximization, Stakeholder Theory, and the Corporate Objective Function. Business Ethics Quarterly, 12 (3), 235-256, 2002.

KOOPMANS, E.: Activity Analysis of Production and Allocation, Cowles Commission for Research in Economics, Monograph $N^{o} 13$. . 1951, New York: Wiley.

KUMBHAKAR, S., LOZANO-VIVAS, A., KNOX, C., \& HASAN, I.: The effects of deregulation on the performance of financial institutions: The case of Spanish Savings Banks. Journal of Money, Credit and Banking , 33 (1), 101-120, 2001.

LEVENE, H.: In Contributions to Probability and Statistics: Essays in Honor of Harold Hotelling, I. Olkin et al. eds. Stanford University Press, 1960.

PASTOR, J.: Eficiencia, cambio productivo y cambio técnico en los bancos y cajas de ahorro españolas: un análisis de la frontera no paramétrico. Revista Española de Economía , 12 (1), 35-73, 1995.

RETOlAZA, J., SAN-JOSE, L., \& RUIZ, M.: Hacia un replanteamiento de la Teoría de Stakeholder: cuatro propuestas innovadoras. En I. Maximsev, V. Krasnoproshin, \& C. Prado, Global Financial \& Business Networks and Information Management Systems. 2010, Minsk-San Petersburg: AEDEM. 
SAN-JOSE, L., RETOLAZA, J., \& GUTIERREZ-GOIRIA, J.: Are Ethical Banks Different? A Comparative Analysis Using the Radical Affinity Index. Journal of Business Ethics, 100 (1), 151-173, 2011.

SEIFORD, L.: Data Envelopment Analysis: The evolution of the state of the art (1978-1995). Journal of Productivity Analysis (7), 99-138, 1996.

SEIFORD, L., \& THRALL, R.: Recent Developments in DEA: The mathematical programming approach to frontier analysis. Journal of Econometrics (46), 7-38, 1990.

TORTOSA-AUSINA, E., GRIFELL-TATJÉ, E., ARMERO, C., \& CONES, D.: Sensitivity analysis of efficiency and Malmquist productivity indices: An application to Spanish savings banks. European Journal of Operational Research , 184 (3), 1062-1084, 2002. 
Anexo 1: Tabla resultados análisis eficiencia.

\begin{tabular}{|c|c|c|c|c|c|c|c|}
\hline EMU & $F P+A T=B=E$ & $F P+A T=B=E+R$ & $\mathrm{FP}+\mathrm{AT}=\mathrm{B}=\mathrm{S}$ & $F P+A T=B=E+B=S$ & $A T=B=E+R$ & $A T=B=S$ & $A T=B=E+B=S$ \\
\hline ALLFUNDS BANK & $99,50 \%$ & $100,00 \%$ & $100,00 \%$ & $100,00 \%$ & $100,00 \%$ & $100,00 \%$ & $100,00 \%$ \\
\hline ALTAE BANCO & $98,10 \%$ & $98,10 \%$ & $61,00 \%$ & $99,20 \%$ & $91,50 \%$ & $37,20 \%$ & $91,80 \%$ \\
\hline ARESBANK & $84,30 \%$ & $84,30 \%$ & $21,60 \%$ & $84,50 \%$ & $84,30 \%$ & $21,60 \%$ & $84,50 \%$ \\
\hline BANCA MARCH & $73,00 \%$ & $73,00 \%$ & $98,10 \%$ & $98,30 \%$ & $73,00 \%$ & $98,10 \%$ & $98,30 \%$ \\
\hline BANCA PUEYO & $93,20 \%$ & $93,20 \%$ & $84,60 \%$ & $99,80 \%$ & $93,20 \%$ & $84,60 \%$ & $97,50 \%$ \\
\hline BANCO ALCALÁ & $100,00 \%$ & $100,00 \%$ & $100,00 \%$ & $100,00 \%$ & $100,00 \%$ & $7,70 \%$ & $100,00 \%$ \\
\hline BANCO BANIF & $83,00 \%$ & $83,00 \%$ & $85,40 \%$ & $90,10 \%$ & $83,00 \%$ & $85,40 \%$ & $90,10 \%$ \\
\hline BANCO BILBAO VIZCAYA ARGENTARIA & $100,00 \%$ & $100,00 \%$ & $100,00 \%$ & $100,00 \%$ & $100,00 \%$ & $100,00 \%$ & $100,00 \%$ \\
\hline BANCO CAIXA GERAL & $71,90 \%$ & $71,90 \%$ & $89,40 \%$ & $92,90 \%$ & $71,90 \%$ & $89,40 \%$ & $92,90 \%$ \\
\hline BANCO CAMINOS & $92,30 \%$ & $92,30 \%$ & $65,10 \%$ & $92,90 \%$ & $92,30 \%$ & $65,10 \%$ & $92,50 \%$ \\
\hline BANCO CETELEM & $100,00 \%$ & $100,00 \%$ & $100,00 \%$ & $100,00 \%$ & $100,00 \%$ & $100,00 \%$ & $100,00 \%$ \\
\hline BANCO COOPERATIVO ESPAÑOL & $76,90 \%$ & $76,90 \%$ & $9,40 \%$ & $79,40 \%$ & $69,10 \%$ & $9,30 \%$ & $69,10 \%$ \\
\hline BANCO DE DEPÓSITOS & $97,70 \%$ & $97,70 \%$ & $29,20 \%$ & $97,70 \%$ & $97,70 \%$ & $28,70 \%$ & $97,70 \%$ \\
\hline BANCO DE FINANZAS E INVERSIONES, & $94,80 \%$ & $94,80 \%$ & $89,90 \%$ & $97,60 \%$ & $94,80 \%$ & $89,90 \%$ & $97,60 \%$ \\
\hline BANCO DE LA PEQUEÑA Y MEDIANA EM & $92,40 \%$ & $92,40 \%$ & $100,00 \%$ & $100,00 \%$ & $91,70 \%$ & $95,90 \%$ & $97,60 \%$ \\
\hline BANCO DE MADRID & $96,50 \%$ & $96,50 \%$ & $96,50 \%$ & $99,10 \%$ & $93,10 \%$ & $96,00 \%$ & $98,60 \%$ \\
\hline BANCO DE SERVICIOS FINANCIEROS C & $73,80 \%$ & $73,80 \%$ & $0,50 \%$ & $73,80 \%$ & $73,80 \%$ & $0,50 \%$ & $73,80 \%$ \\
\hline BANCO DE VALENCIA & $68,20 \%$ & $68,20 \%$ & $99,20 \%$ & $99,20 \%$ & $68,00 \%$ & $99,20 \%$ & $99,20 \%$ \\
\hline BANCO DEPOSITARIO BBVA & $97,30 \%$ & $97,60 \%$ & $6,10 \%$ & $97,70 \%$ & $97,00 \%$ & $5,60 \%$ & $97,00 \%$ \\
\hline BANCO ESPAÑOL DE CRÉDITO & $76,80 \%$ & $76,80 \%$ & $90,90 \%$ & $100,00 \%$ & $64,00 \%$ & $82,30 \%$ & $87,20 \%$ \\
\hline BANCO ETCHEVERRÍA & $98,50 \%$ & $98,50 \%$ & $96,20 \%$ & $100,00 \%$ & $95,20 \%$ & $85,90 \%$ & $97,70 \%$ \\
\hline BANCO EUROPEO DE FINANZAS & $98,70 \%$ & $98,70 \%$ & $15,20 \%$ & $98,70 \%$ & $98,70 \%$ & $15,20 \%$ & $98,70 \%$ \\
\hline BANCO FINANTIA SOFINLOC & $99,40 \%$ & $99,40 \%$ & $28,50 \%$ & $99,50 \%$ & $99,40 \%$ & $19,50 \%$ & $99,50 \%$ \\
\hline BANCO GALLEGO & $73,70 \%$ & $73,70 \%$ & $100,00 \%$ & $100,00 \%$ & $73,30 \%$ & $100,00 \%$ & $100,00 \%$ \\
\hline BANCO GUIPUZCOANO & $71,90 \%$ & $71,90 \%$ & $80,90 \%$ & $85,70 \%$ & $71,90 \%$ & $80,90 \%$ & $85,70 \%$ \\
\hline BANCO HALIFAX HISPANIA & $92,60 \%$ & $92,60 \%$ & $100,00 \%$ & $100,00 \%$ & $81,80 \%$ & $100,00 \%$ & $100,00 \%$ \\
\hline BANCO INVERSIS & $91,30 \%$ & $91,30 \%$ & $93,80 \%$ & $98,80 \%$ & $91,30 \%$ & $93,80 \%$ & $98,80 \%$ \\
\hline BANCO PASTOR & $73,60 \%$ & $73,60 \%$ & $85,90 \%$ & $99,90 \%$ & $68,30 \%$ & $84,20 \%$ & $91,00 \%$ \\
\hline BANCO POPULAR ESPAÑOL & $59,60 \%$ & $59,60 \%$ & $92,00 \%$ & $93,00 \%$ & $59,60 \%$ & $92,00 \%$ & $93,00 \%$ \\
\hline BANCO POPULAR HIPOTECARIO & $80,70 \%$ & $80,70 \%$ & $94,20 \%$ & $94,20 \%$ & $80,70 \%$ & $94,20 \%$ & $94,20 \%$ \\
\hline BANCO SABADELL & $69,60 \%$ & $69,60 \%$ & $100,00 \%$ & $100,00 \%$ & $69,60 \%$ & $100,00 \%$ & $100,00 \%$ \\
\hline BANCO SANTANDER & $70,40 \%$ & $70,40 \%$ & $76,50 \%$ & $76,50 \%$ & $70,40 \%$ & $76,50 \%$ & $76,50 \%$ \\
\hline BANCO URQUIJO SABADELL BANCA PRI & $83,50 \%$ & $83,50 \%$ & $58,20 \%$ & $90,10 \%$ & $83,50 \%$ & $58,20 \%$ & $90,10 \%$ \\
\hline BANCOFAR & $91,40 \%$ & $91,40 \%$ & $89,50 \%$ & $93,40 \%$ & $87,30 \%$ & $89,50 \%$ & $91,80 \%$ \\
\hline BANCOPOPULAR-E & $93,10 \%$ & $93,10 \%$ & $92,30 \%$ & $96,50 \%$ & $92,30 \%$ & $92,30 \%$ & $96,50 \%$ \\
\hline BANKINTER & $80,50 \%$ & $80,50 \%$ & $92,20 \%$ & $100,00 \%$ & $72,30 \%$ & $90,90 \%$ & $94,00 \%$ \\
\hline BANKOA & $87,00 \%$ & $87,00 \%$ & $92,20 \%$ & $96,10 \%$ & $85,50 \%$ & $92,20 \%$ & $96,10 \%$ \\
\hline BARCLAYS BANK & $50,90 \%$ & $50,90 \%$ & $97,80 \%$ & $97,80 \%$ & $43,60 \%$ & $92,60 \%$ & $92,60 \%$ \\
\hline BBK & $81,70 \%$ & $81,70 \%$ & $89,20 \%$ & $94,90 \%$ & $81,70 \%$ & $89,20 \%$ & $94,90 \%$ \\
\hline BNP PARIBAS ESPAÑA & $94,80 \%$ & $94,80 \%$ & $92,50 \%$ & $99,30 \%$ & $94,80 \%$ & $92,50 \%$ & $98,80 \%$ \\
\hline Bancaja & $58,50 \%$ & $58,50 \%$ & $100,00 \%$ & $100,00 \%$ & $50,20 \%$ & $97,50 \%$ & $97,50 \%$ \\
\hline CITIBANK ESPAÑA & $62,30 \%$ & $62,30 \%$ & $97,60 \%$ & $97,60 \%$ & $62,30 \%$ & $96,30 \%$ & $96,30 \%$ \\
\hline Caixa Catalunya & $68,20 \%$ & $68,20 \%$ & $100,00 \%$ & $100,00 \%$ & $63,80 \%$ & $100,00 \%$ & $100,00 \%$ \\
\hline Caixa Galicia & $65,20 \%$ & $65,20 \%$ & $100,00 \%$ & $100,00 \%$ & $58,20 \%$ & $98,80 \%$ & $98,80 \%$ \\
\hline Caixa Girona & $80,30 \%$ & $80,30 \%$ & $88,20 \%$ & $98,00 \%$ & $78,90 \%$ & $88,20 \%$ & $95,90 \%$ \\
\hline Caixa Laietana & $77,70 \%$ & $77,70 \%$ & $90,90 \%$ & $91,70 \%$ & $76,10 \%$ & $90,80 \%$ & $91,10 \%$ \\
\hline Caixa Manresa & $75,60 \%$ & $75,60 \%$ & $81,50 \%$ & $90,20 \%$ & $74,20 \%$ & $81,50 \%$ & $87,90 \%$ \\
\hline Caixa Ontinyent & $94,30 \%$ & $94,30 \%$ & $100,00 \%$ & $100,00 \%$ & $91,70 \%$ & $100,00 \%$ & $100,00 \%$ \\
\hline Caixa Penedés & $68,40 \%$ & $68,40 \%$ & $91,60 \%$ & $96,30 \%$ & $64,40 \%$ & $90,30 \%$ & $91,30 \%$ \\
\hline Caixa Sabadell & $74,80 \%$ & $74,80 \%$ & $84,80 \%$ & $95,40 \%$ & $72,10 \%$ & $84,10 \%$ & $91,80 \%$ \\
\hline Caixa Tarragona & $80,40 \%$ & $80,40 \%$ & $76,80 \%$ & $97,80 \%$ & $77,90 \%$ & $76,30 \%$ & $94,40 \%$ \\
\hline Caixa Terrassa & $84,00 \%$ & $84,00 \%$ & $80,20 \%$ & $100,00 \%$ & $80,40 \%$ & $78,60 \%$ & $93,90 \%$ \\
\hline Caixa de Manlleu & $87,10 \%$ & $87,10 \%$ & $100,00 \%$ & $100,00 \%$ & $79,00 \%$ & $100,00 \%$ & $100,00 \%$ \\
\hline Caixanova & $62,70 \%$ & $62,70 \%$ & $85,00 \%$ & $90,70 \%$ & $61,30 \%$ & $84,40 \%$ & $87,40 \%$ \\
\hline Caja Cantabria & $77,00 \%$ & $77,00 \%$ & $87,60 \%$ & $89,20 \%$ & $75,20 \%$ & $87,30 \%$ & $88,40 \%$ \\
\hline Caja Círculo de Burgos & $73,70 \%$ & $73,70 \%$ & $79,50 \%$ & $88,60 \%$ & $73,70 \%$ & $79,50 \%$ & $88,60 \%$ \\
\hline Caja Duero & $69,30 \%$ & $69,30 \%$ & $81,20 \%$ & $92,20 \%$ & $69,30 \%$ & $81,20 \%$ & $91,30 \%$ \\
\hline Caja España & $68,20 \%$ & $68,20 \%$ & $82,40 \%$ & $97,20 \%$ & $63,10 \%$ & $80,80 \%$ & $88,00 \%$ \\
\hline Caja Extremadura & $77,10 \%$ & $77,10 \%$ & $89,80 \%$ & $97,10 \%$ & $77,10 \%$ & $89,80 \%$ & $97,10 \%$ \\
\hline Caja General de Canarias & $70,60 \%$ & $70,60 \%$ & $80,80 \%$ & $86,40 \%$ & $70,60 \%$ & $80,80 \%$ & $86,40 \%$ \\
\hline Caja Granada & $67,90 \%$ & $67,90 \%$ & $100,00 \%$ & $100,00 \%$ & $67,90 \%$ & $100,00 \%$ & $100,00 \%$ \\
\hline Caja Inmaculada & $75,30 \%$ & $75,30 \%$ & $84,10 \%$ & $89,10 \%$ & $75,30 \%$ & $84,10 \%$ & $89,10 \%$ \\
\hline Caja Insular Canarias & $74,70 \%$ & $74,70 \%$ & $80,70 \%$ & $89,20 \%$ & $72,50 \%$ & $80,70 \%$ & $86,60 \%$ \\
\hline Caja Madrid & $48,80 \%$ & $48,80 \%$ & $84,50 \%$ & $84,90 \%$ & $48,80 \%$ & $84,50 \%$ & $84,90 \%$ \\
\hline Caja Mediterráneo & $69,60 \%$ & $69,60 \%$ & $97,50 \%$ & $100,00 \%$ & $61,80 \%$ & $93,90 \%$ & $93,90 \%$ \\
\hline Caja Murcia & $79,50 \%$ & $79,50 \%$ & $82,90 \%$ & $91,50 \%$ & $79,50 \%$ & $82,90 \%$ & $91,20 \%$ \\
\hline Caja Navarra & $70,40 \%$ & $70,40 \%$ & $82,30 \%$ & $85,60 \%$ & $70,40 \%$ & $82,30 \%$ & $85,60 \%$ \\
\hline Caja Rioja & $76,00 \%$ & $76,00 \%$ & $82,50 \%$ & $90,20 \%$ & $75,40 \%$ & $82,50 \%$ & $88,80 \%$ \\
\hline Caja Segovia & $79,70 \%$ & $79,70 \%$ & $76,90 \%$ & $86,50 \%$ & $79,00 \%$ & $76,90 \%$ & $85,70 \%$ \\
\hline Caja Sol & $61,50 \%$ & $61,50 \%$ & $100,00 \%$ & $100,00 \%$ & $61,50 \%$ & $100,00 \%$ & $100,00 \%$ \\
\hline Caja Sur & $52,50 \%$ & $52,50 \%$ & $100,00 \%$ & $100,00 \%$ & $41,30 \%$ & $99,60 \%$ & $99,60 \%$ \\
\hline Caja de Badajoz & $75,80 \%$ & $75,80 \%$ & $100,00 \%$ & $100,00 \%$ & $75,80 \%$ & $100,00 \%$ & $100,00 \%$ \\
\hline
\end{tabular}




\begin{tabular}{|c|c|c|c|c|c|c|c|}
\hline Caja de Burgos & $77,60 \%$ & $77,60 \%$ & $76,90 \%$ & $83,10 \%$ & $77,60 \%$ & $76,90 \%$ & $83,10 \%$ \\
\hline Caja de Guadalajara & $93,60 \%$ & $93,60 \%$ & $88,50 \%$ & $99,10 \%$ & $85,50 \%$ & $85,50 \%$ & $94,70 \%$ \\
\hline Caja de Jaén & $94,00 \%$ & $94,00 \%$ & $85,80 \%$ & $99,60 \%$ & $91,70 \%$ & $85,00 \%$ & $96,60 \%$ \\
\hline Caja de Ávila & $75,90 \%$ & $75,90 \%$ & $77,00 \%$ & $83,70 \%$ & $75,30 \%$ & $77,00 \%$ & $83,10 \%$ \\
\hline Cajastur & $84,00 \%$ & $84,00 \%$ & $82,80 \%$ & $97,00 \%$ & $84,00 \%$ & $82,80 \%$ & $97,00 \%$ \\
\hline Colonya Caixa Pollença & $99,20 \%$ & $99,20 \%$ & $100,00 \%$ & $100,00 \%$ & $97,50 \%$ & $96,60 \%$ & $99,50 \%$ \\
\hline DEUTSCHE BANK S.A.E. & $72,60 \%$ & $72,60 \%$ & $100,00 \%$ & $100,00 \%$ & $70,50 \%$ & $100,00 \%$ & $100,00 \%$ \\
\hline DEXIA SABADELL & $82,90 \%$ & $82,90 \%$ & $86,30 \%$ & $100,00 \%$ & $73,00 \%$ & $78,20 \%$ & $82,80 \%$ \\
\hline EBN BANCO DE NEGOCIOS & $94,30 \%$ & $94,30 \%$ & $53,70 \%$ & $94,50 \%$ & $94,30 \%$ & $53,70 \%$ & $94,50 \%$ \\
\hline FINANZIA, BANCO DE CRÉDITO & $60,50 \%$ & $60,50 \%$ & $79,70 \%$ & $80,20 \%$ & $55,00 \%$ & $72,90 \%$ & $72,90 \%$ \\
\hline GENERAL ELECTRIC CAPITAL BANK & $69,80 \%$ & $69,80 \%$ & $99,70 \%$ & $100,00 \%$ & $69,80 \%$ & $99,70 \%$ & $100,00 \%$ \\
\hline Ibercaja & $66,80 \%$ & $66,80 \%$ & $99,20 \%$ & $99,30 \%$ & $66,80 \%$ & $99,20 \%$ & $99,30 \%$ \\
\hline Kutxa & $76,40 \%$ & $76,40 \%$ & $93,00 \%$ & $99,50 \%$ & $76,40 \%$ & $93,00 \%$ & $99,50 \%$ \\
\hline La Caixa & $56,70 \%$ & $56,70 \%$ & $100,00 \%$ & $100,00 \%$ & $52,70 \%$ & $100,00 \%$ & $100,00 \%$ \\
\hline MICROBANK DE LA CAIXA & $99,80 \%$ & $99,80 \%$ & $100,00 \%$ & $100,00 \%$ & $99,80 \%$ & $100,00 \%$ & $100,00 \%$ \\
\hline OPEN BANK SANTANDER CONSUMER & $89,90 \%$ & $89,90 \%$ & $17,70 \%$ & $91,50 \%$ & $74,00 \%$ & $15,10 \%$ & $75,80 \%$ \\
\hline POPULAR BANCA PRIVADA & $96,10 \%$ & $96,10 \%$ & $45,20 \%$ & $98,10 \%$ & $84,90 \%$ & $35,20 \%$ & $88,60 \%$ \\
\hline PRIVAT BANK DEGROOF & $100,00 \%$ & $100,00 \%$ & $100,00 \%$ & $100,00 \%$ & $100,00 \%$ & $100,00 \%$ & $100,00 \%$ \\
\hline RBC DEXIA INVESTOR SERVICES ESPA & $89,90 \%$ & $89,90 \%$ & $28,80 \%$ & $91,70 \%$ & $80,70 \%$ & $25,80 \%$ & $84,70 \%$ \\
\hline SANTANDER CONSUMER FINANCE & $96,30 \%$ & $96,30 \%$ & $24,40 \%$ & $96,30 \%$ & $96,30 \%$ & $24,40 \%$ & $96,30 \%$ \\
\hline SANTANDER INVESTMENT & $92,00 \%$ & $92,00 \%$ & $35,00 \%$ & $92,00 \%$ & $85,60 \%$ & $32,20 \%$ & $85,60 \%$ \\
\hline SELF TRADE BANK & $98,50 \%$ & $98,50 \%$ & $48,30 \%$ & $98,60 \%$ & $98,50 \%$ & $48,30 \%$ & $98,60 \%$ \\
\hline Sa nostra & $56,40 \%$ & $56,40 \%$ & $87,90 \%$ & $87,90 \%$ & $54,00 \%$ & $86,20 \%$ & $86,20 \%$ \\
\hline UBS BANK & $88,40 \%$ & $88,40 \%$ & $86,60 \%$ & $95,50 \%$ & $88,40 \%$ & $84,50 \%$ & $92,90 \%$ \\
\hline UNO-E BANK & $82,30 \%$ & $82,30 \%$ & $78,30 \%$ & $82,40 \%$ & $82,30 \%$ & $78,30 \%$ & $82,40 \%$ \\
\hline Unicaja & $68,20 \%$ & $68,20 \%$ & $94,10 \%$ & $98,80 \%$ & $68,20 \%$ & $94,10 \%$ & $98,80 \%$ \\
\hline Vital Kutxa & $78,40 \%$ & $78,40 \%$ & $84,60 \%$ & $86,90 \%$ & $78,40 \%$ & $84,60 \%$ & $86,90 \%$ \\
\hline
\end{tabular}

